\title{
The thermal imaging parameters in correlation with USG duplex parameters used in chronic venous disease of lower extremities diagnosis
}

\author{
by J. Kajewska*, A. Cholewka*, J. Pająk*, K. Sieroń - Stołtny**, Z. Drzazga*, \\ M. Kawecki ${ }^{* * *}$ and Agata Stanek ${ }^{* * * *}$
}

\begin{abstract}
* University of Silesia, 40-007, Bankowa Str., Katowice, Poland, armand.cholewka@us.edu.pl
${ }^{*}$ School of Health Sciences in Katowice, Department of Physical Medicine, Chair of Physiotherapy, Medical University of Silesia, Medyków Street 12, 40-752 Katowice, Poland

${ }^{* * *}$ Burn Treatment Center, Siemianowice Slaskie, Poland

${ }^{* * * *}$ School of Medicine with the Division of Dentistry in Zabrze, Department and Clinic of Internal Diseases, Angiology and Physical Medicine in Bytom, Medical University of Silesia, Batorego Street 15, 41-902 Bytom, Poland
\end{abstract}

\begin{abstract}
The aim of this study was to demonstrate the possibilities of using the thermal imaging in lower extremities chronic venous disease (CVD) diagnosis and evaluation parameters like: the degree and range of reflux. The study was conducted on a group of patients with CVD and healthy subjects. Obtained results showed that thermal imaging may give not only qualitative but also quantitative parameters what was confirmed by statistical correlation (i.e. for isotherm Area vs reflux where correlation coefficient $r=0,46)$. Obtained results showed the possibilities for the use of thermal imaging in clinical diagnosis of lower extremities superficial veins disorders.
\end{abstract}

\section{Introduction}

Nowadays in the Western industrialized countries, chronic venous disease (CVD) is one of the most common public health problems. CVD occurs in about 40 to $50 \%$ of the male population and $50-55 \%$ of women [1]. In the US alone, it has been estimated that as many as $23 \%$ of the adult population suffer from varicose veins, while $6 \%$ have more advanced chronic venous disease including skin changes or active venous ulcers [2]. Sometimes the CVDs are considered by the patients only as a cosmetic defect and not a disease, even though they may result in pain, discomfort, disability or reduced quality of life [3,4]. That's why CVD is not only medical but also social problem. It is also called a civilization disease. Therefore there is a need to find a simple, quite cheap and fast method for physicians, which in a quick and easy way would show a pathology of the venous system allowing the physician to refer the patient to a proper specialist.

Duplex ultrasound, a leading and standard method used in CVD diagnosis, may not show all pathology changes connected with veins of lower limb at an early stage of CVD and may be unreliable in some patients when the venous disease is related to an impairment of the microcirculation. That is why it is very important to find a new diagnosis technique that can bring some parameters describing not only structural changes but also early metabolic ones. That is why angiologists, but first of all general practitioners need easy and non-invasive technique that allows imaging of CVDs in the very early stadium what may point out at thermovision.

\section{Materials and methods}

The research group consisted of 16 patients ( 11 women and 5 men) suffering from primary chronic venous disease. There was also control group consisting of 9 healthy people (6 women and 3 men). Each patient as well as all volunteers in the control group have been examined by a duplex ultrasound. It was necessary to confirm the duplex parameters in patients and to exclude the presence of pathology in lower extremity venous system in the case of healthy volunteers. 


\subsection{1/qirt.2016.049}

The enrolment for the trial was performed in a group of patients with primary chronic disease stages C2 - C4 who did not suffer from any other diseases, and in duplex scanning reflux duration $>500 \mathrm{~ms}$ in the great saphenous vein (GSV) the only deviation was found.

The investigations were carried out at the Chair and Clinic of Internal Diseases and Physical Medicine, Silesian Medical University in Bytom, Poland.

All patients before the study were examined by a physician. They were requested not to smoke, drink alcohol or hot drinks for at least three hours before the thermovision studies.

The thermal imaging of the lower extremities skin surface was performed with use of a Thermovision Camera E60 by Flir Systems, Sweden. All patients were diagnosed by duplex scanning (device Logic7) and the reflux duration as well as the range of reflux were determined.

Thermal imaging were performed in a special room, where the temperature was stabilized and was close to $23 \pm 1{ }^{\circ} \mathrm{C}$. The distance between the infrared camera and the patient during the examination was $1-1,5 \mathrm{~m}$, depending on the size of the limbs. It was necessary to follow the standard protocol of infrared imaging in medicine $[5,7]$.

For the study the following parameters were taken into account:

- mean venous reflux duration(VR), counted by duplex scan

- $\quad$ range of vein insufficiency (Range), counted by duplex scan

- mean temperature derived from isotherm area $\left(\mathbf{T}_{\text {iso.area}}\right)$. Isotherm areas were marked using the mean temperature obtained for the whole lower limb of the healthy volunteers group. The temperature above the mean limb temperature was taken as a temperature threshold used for the isotherm area in respective thermograms.

- $\quad$ mean temperature of lower extremity $\left(\mathrm{T}_{\text {l.ext. }}\right)$, counted from thermograms

- $\quad$ ratio of whole lower extremity area and isotherm area (Area\%).

All the volunteers underwent the procedure of thermal imaging of lower limbs in similar regions as the patients. Each patient signed a written consent for the diagnostics form, and they were previously informed about the plans and procedures of the trial.

Thermograms were analysed by the use of a ThermaCAM TM Researcher Pro 2.8 SR-3 and the statistical analysis was done in Statistica software.

Thermal imaging and ultrasound Doppler examination was performed on each group and the analysis of obtained thermal parameters data was carried out as follows.

Isotherm areas were marked on the whole lower extremities surface using the mean temperature of the lower limb from control group (in practice it was sum of anterior area and posterior limb area). The temperature higher than the mean limb temperature of control group was taken as a temperature threshold and made the isotherm area in respective thermograms. Such obtained areas were used to evaluate the percentage ratio between isotherm area and whole limb area but the threshold temperature was derived from the healthy people unlike to first method where it was equal to mean temperature of examined limb.

\section{Results and Discussion}

Skin temperature is an indicator of the heat exchange between internal tissues and the environment in which the organism is located. This fact is illustrated in the thermogram of the representative object in Figure 1. Superficial vein diseases seem to be a very interesting and pertinent issue for thermal imaging.

Obtained patients thermograms shown elongated areas characterizing tracks of the great saphenous vein with a higher temperature which can be connected with pathological changes in the veins. The increase of temperature in some areas of the thighs seems to be connected with blood stasis due to a malfunction in the vein's valves. Such processes can cause strain to the veins and lead to inflammation that causes increase of temperature in this area [6]. These areas are characterized by higher temperatures in opposition to the normal lower limb thermal image (Fig. 3). It appears that the differences in the temperature gradient can be easy correlated with the medical diagnosis.

For example Figure 1a shows thermal image of the patient with great saphenous vein insufficiency and with an inflammatory state in the surrounding soft tissues what can be seen even on the lower limb. Figures $1 \mathrm{~b}$ presents thermograms for two different patients suffering from great saphenous vein insufficiency. 

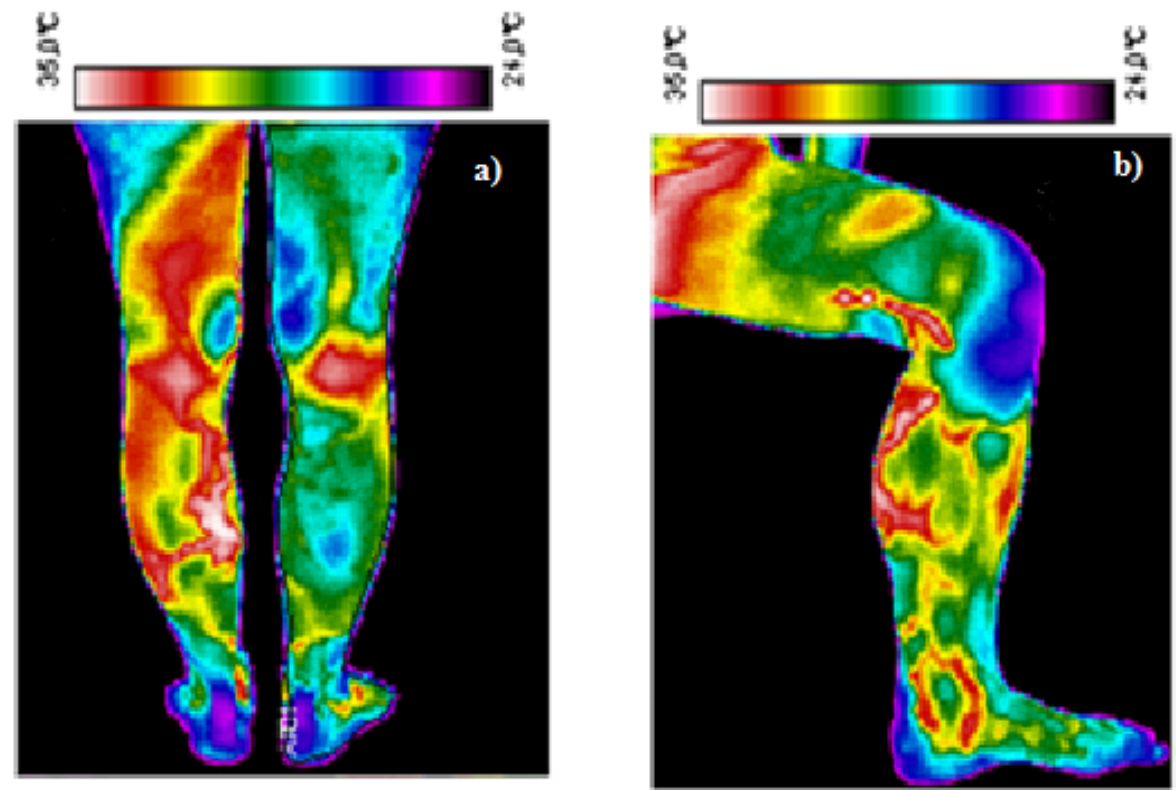

Fig. 1 The thermograms of the inside limb parts suffering from an insufficiency in the great saphenous vein of the lower left limb

It is easy to see on the thermal images that the areas characterized with higher temperature are connected with disease changes. Moreover it can be underlined that using isotherm function and isotherm areas may give another way to analysis thermgrams what was presented in Figure 2. In further part of the work such isotherm areas will be taken into consideration in comparison to the Reflux counted from the dupplex.
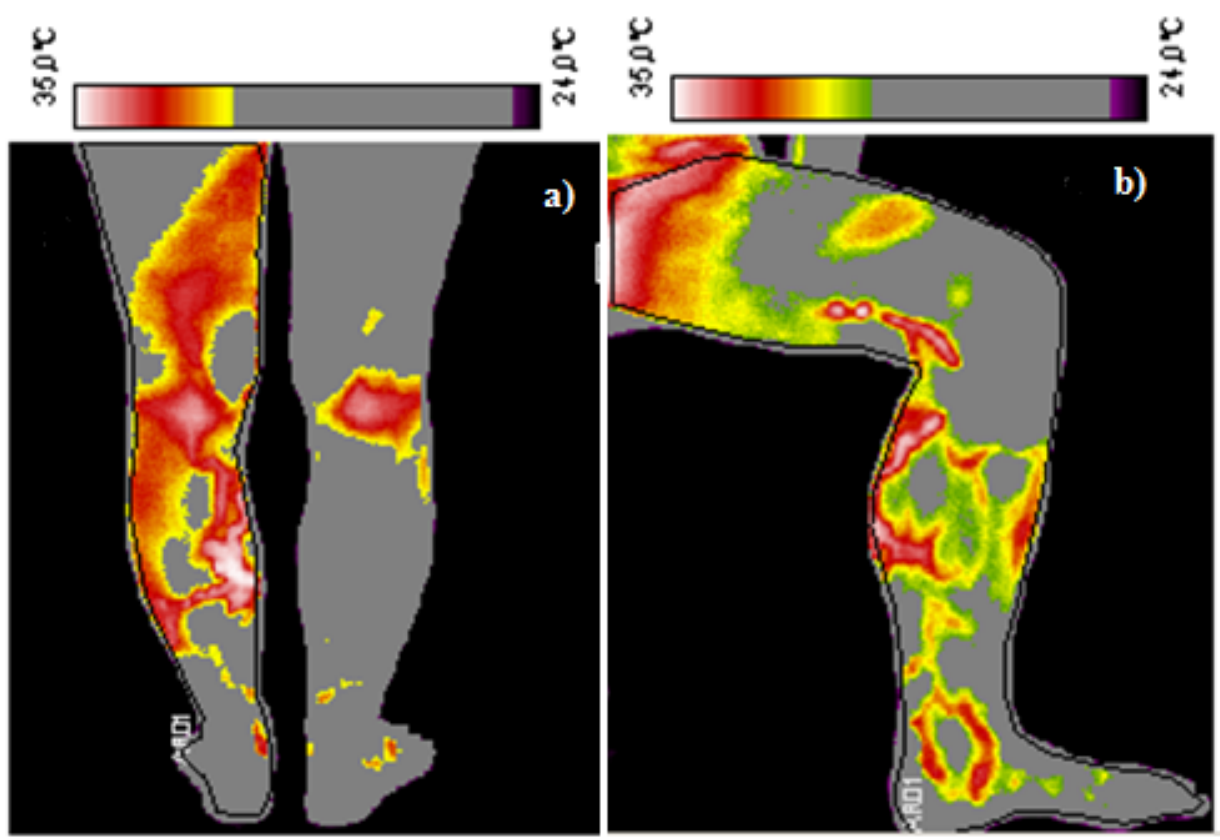

Fig. 2 The thermograms front of the lower limbs of the patient showing the interested area after subtracting the isotherm

The thermogram presented in Figure 1a reveals a prolonged area on the left limb in posterior view ending below the dół kolanowy. It may be correlated with the great spaphena vein insufficiency and the inflammatory state of soft tissues around it, which occurred in this patient. Similar thermal map is seen in Figure $1 \mathrm{~b}$ which reveals longer areas 


\subsection{1/qirt.2016.049}

characterized with a higher temperature for patient suffering from vena saphena magna insufficiency. However, it is seen that the areas revealed on the skin are longer and more focused in the tracks of the veins. An elongated area could be seen passing through the left limb from the thigh to almost ankle. This is probably associated with a range of venous insufficiency what was also one of the aims in presented paper.
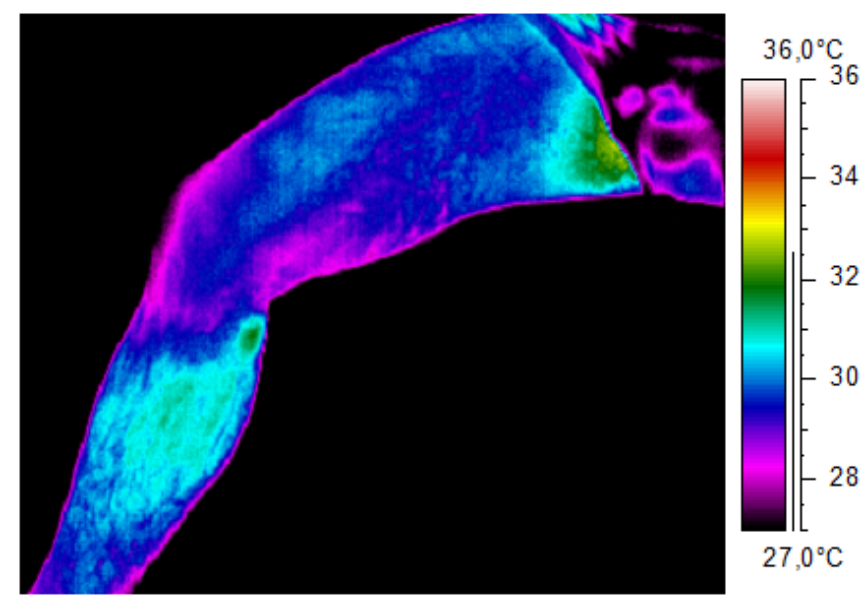

\begin{tabular}{cc}
\hline Area & Temperature $^{\circ} \mathbf{C}$ \\
\hline
\end{tabular}

Right lower

limb: $\mathrm{T}_{\text {mean }}$

Fig. 3 Thermograms of inside limb parts representative for healthy group

For a deeper insight into the problem the statistical analysis was performed. The differences between the mean temperature of the whole lower extremities for patients with chronic venous diseases vein insufficiency and the healthy ones were compared (Figure 4).

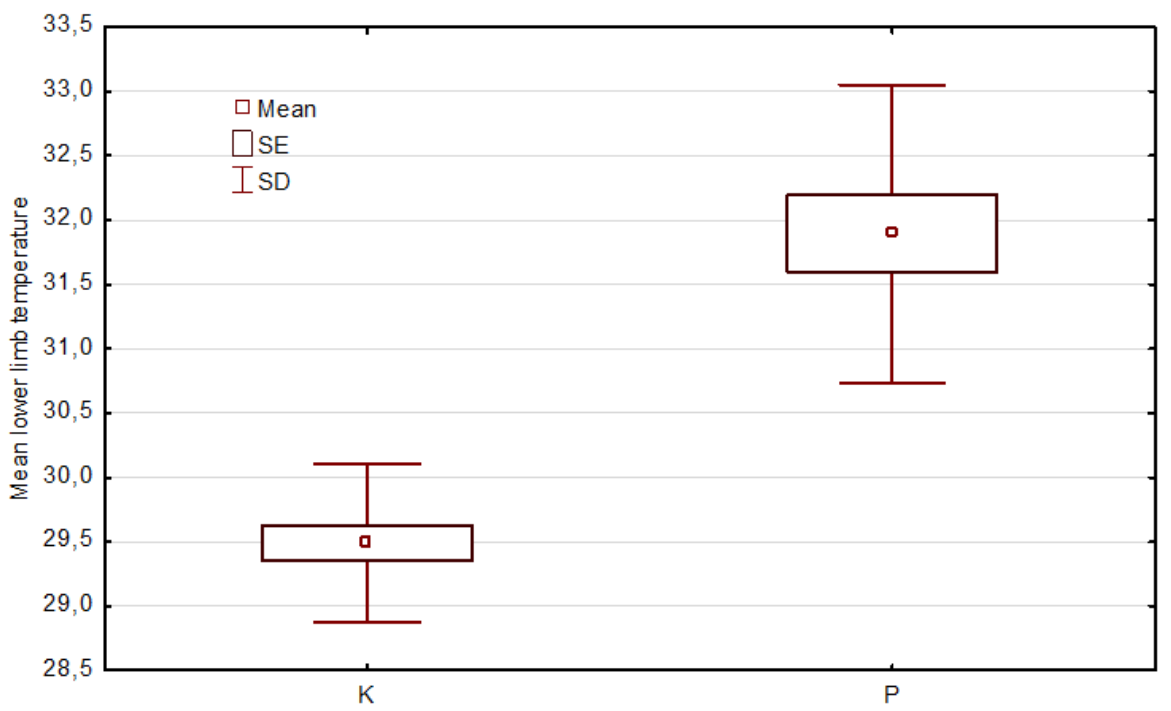

Fig. 4. The mean temperature of the lower extremities shows the differences obtained between healthy and patients with vein insufficiency. Statistical values used: mean, SE and SD, mean value of temperature, standard error and standard deviation. 


\subsection{1/qirt.2016.049}

All parameters used in the statistical analysis and correlation were collected in Table 2.

\begin{tabular}{|c|c|c|c|c|c|c|c|}
\hline Patient number & VR $[\mathbf{s}]$ & Range & $\mathbf{T}_{\text {iro.area }}$ & $\mathbf{T}_{1 \_ \text {extr. }}$ & Thermal Range & $\boldsymbol{\Delta} \mathbf{T}$ & (Izo.Area)/(1_extr. Area) [\%] \\
\hline \multirow{2}{*}{1} & 3 & 4 & 31,4 & 32,5 & 0,95 & 1,1 & 54 \\
\cline { 2 - 8 } & 2 & 4 & 31,5 & 32,4 & 0,93 & 0,9 & 59 \\
\hline 2 & 3 & 1 & 33,1 & 34 & 0,89 & 0,9 & 53 \\
\hline 3 & 3 & 3 & 31,4 & 32,1 & 0,89 & 0,7 & 49 \\
\hline 4 & 2 & 3 & 31,7 & 32,7 & 0,83 & 1 & 48 \\
\hline
\end{tabular}

Tab. 1The temperature parameters (mean temperature derived from isotherm area ( $\left.T_{\text {iso.areaa }}\right)$, mean temperature of lower extremity ( $\left.T_{\text {l.ext. }}\right)$, range of isotherm area (Thermal range), temperature difference $\left(\Delta T=T_{\text {iso.area- }} T_{\text {l.ext }}\right)$, ratio of whole lower extremity area and isotherm area (Area\%) and duplex parameters (mean venous reflux diuration (VR), range of vein insufficiency (Range) obtained from the sample group of patients

To find out if thermal imaging may be useful in chronic venous disorders it was necessary to check the correlation between parameters obtained with duplex ultrasound and thermal parameters derived from the thermograms. The results of this analysis are presented in Fig. 5-7).

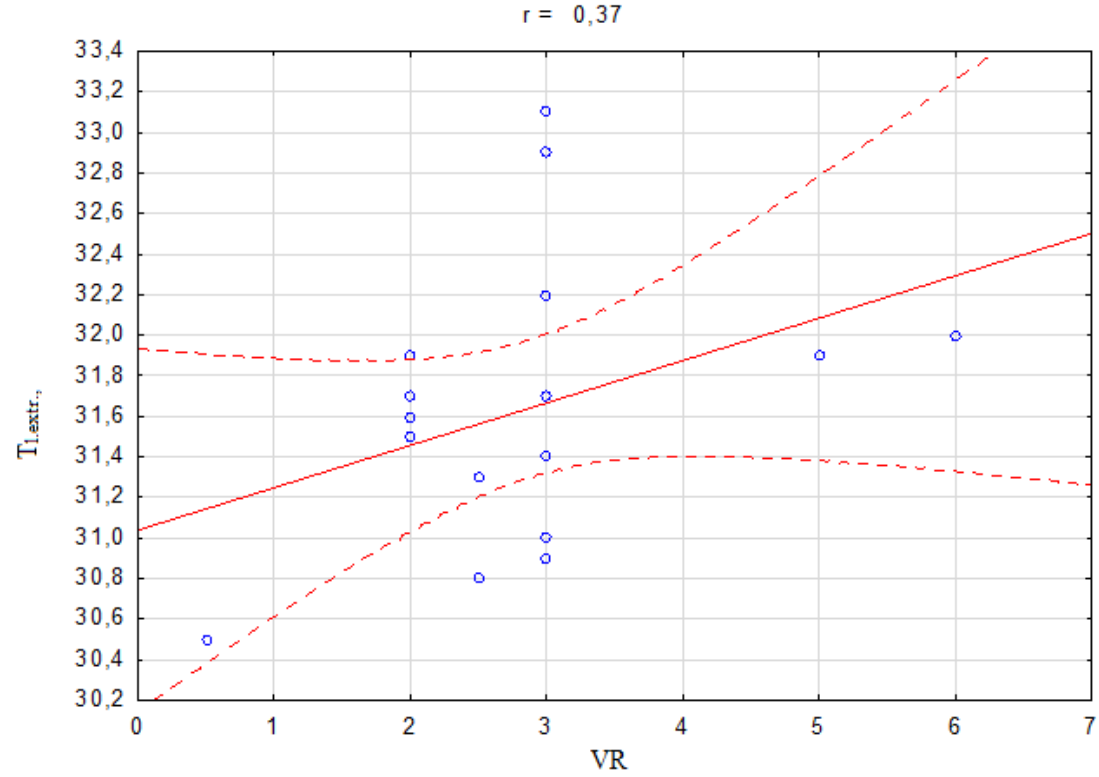

Fig 5. Scatterplot between Lower Limb mean temperature (Tl.extr., ) and mean Venous Reflux Diuration obtained for all studied patients, where $r$ is the correlation coefficient.

One can see in the Figure 4 that Reflux, which is backflow of blood due to wrong operating venous valves, is described by average positive dependence on the average temperature of the limb. This means that the measure of the average temperature of the patient's limb can give some useful information. The average temperature related to the state of health of the peripheral circulation of the lower limb and the information obtained this way with thermal imaging can direct the doctor for further diagnostic procedures. 


\subsection{1/qirt.2016.049}

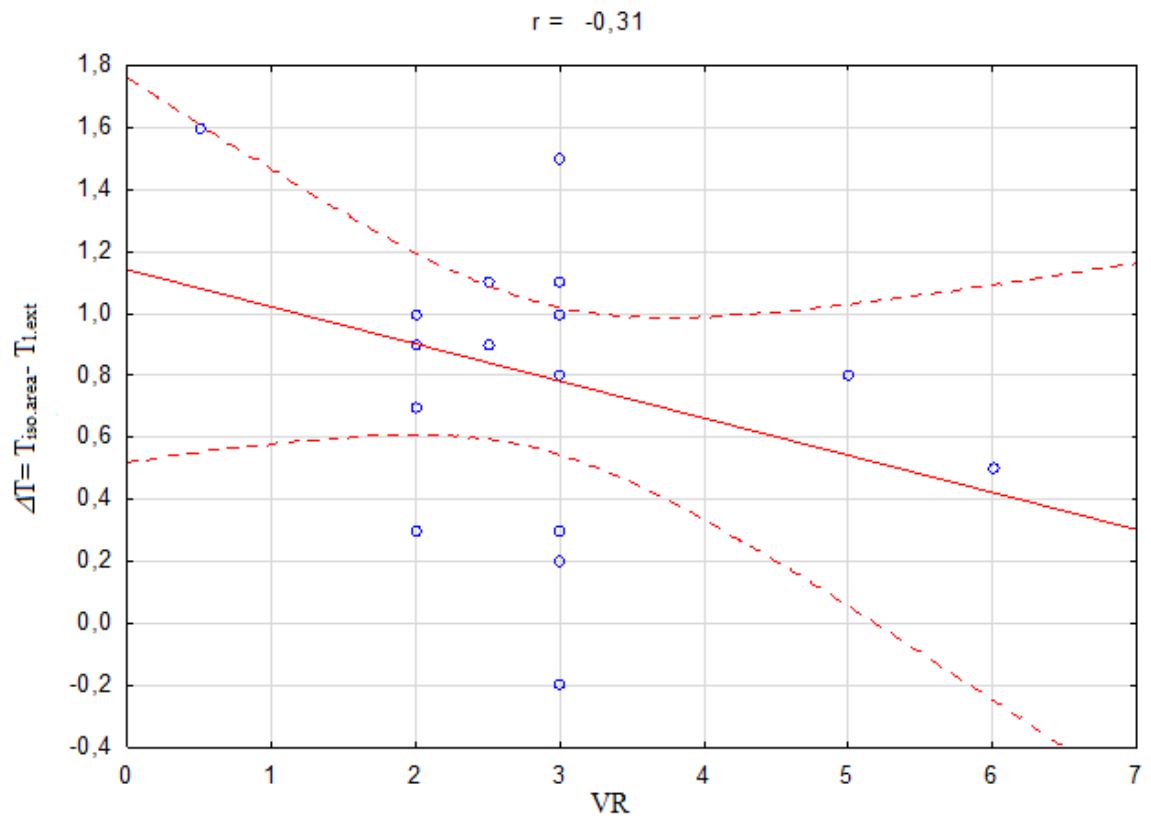

Fig. 6. Scatterplot between mean Venous Reflux Diuration (VR) and difference between mean temperature of isotherm area (Tiso.area, ) and mean temperature of whole lower limb for all studied patients, where $r$ is the correlation coefficient.

The resulting average negative correlation between VR and the difference in average temperature of the isotherm and the mean temperature of the whole lower extremity may suggest that this difference is higher for patients who suffer from relatively small ranges of CVD. Thus, the average temperature of the area bounded by the isotherm to the average temperature value of the entire (entire area is calculated as the sum of the front and back) limb ratio may also carry some information to the physician. It is possibility that such parameter may be a threshold at screening, however, the resulting values should be validated and tested for a larger population of patients

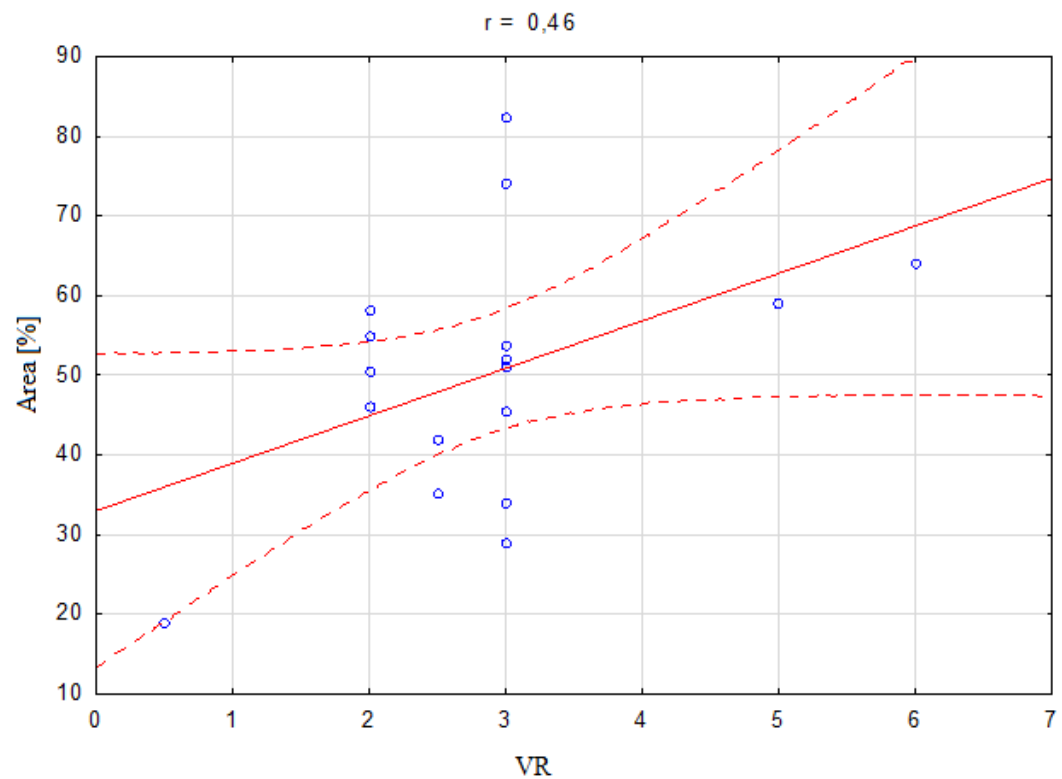

Fig 6. Scatterplot obtained for mean Venous Reflux Diuration and ratio of isotherm area to whole lower extremity area (Area\%) obtained from all patients, where $r$ is correlation coefficient. 


\subsection{1/qirt.2016.049}

The greatest diagnostic expectations can be associated with a parameter that is related to the isotherm area (isothermal threshold value is the average temperature of the limbs obtained for healthy volunteers) and area of the entire patient's limb ratio. The resulting average positive correlation between this ratio $(r=0.46)$ and Venous Reflux Diuration indicates that the thermal diagnostic may find potential use in clinical diagnosis or screening. However, as for the other parameters proposed in this paper, received values should be validated and tested to a much larger population of patients.

Presented graphs suggest that there is a connection between the thermal map and derived from them parameters and duplex parameters obtained for patients suffered from great saphenous vein insufficiency. The inflammatory state in the surrounding of soft tissues manifests as a areas characterized by higher temperature what make them clearly visible, owing to changes as a result of disease - inflammatory states connected with vein insufficiency.

It seems that the use of thermal imaging as a diagnostic or screening method for identifying venous diseases appears to be very promising. This allows to obtain thermal images that are correlated with the condition of veins and small blood vessel circulation. In addition, the infrared images can reveal many elements that are not visible in Doppler ultrasound. Thermal imaging is used as a technique that can show the metabolism and functional changes manifested as changes in the skin temperature. However, each patient should be taken into consideration individually. It is very important to evaluate a standard protocol for the use of thermography for CVD.

\section{Conclusions}

Thermal imaging as a completely non-invasive technique can be helpful in the diagnosis of superficial vein insufficiency. Obtained results showed that thermal imaging may give not only qualitative but also quantitative parameters what was confirmed by correlations between thermal and duplex ultrasound parameters.

It is possible that thermovision may be first diagnosis technique used as a screening method in patients with some lower extremities veins disordes.

However, this requires further research on a larger group of studied patients.

\section{REFERENCES}

[1] Van den Oever R, Hepp B, Debbaut B, Simon I (1998) Socio-economic impact of chronic venous insufficiency. An underestimated public health problem. Int Angiol 17:161-167.

[2] Kaplan RM, Criqui MH, Denenberg JO, Bergan J, Fronek A (2003) Quality of life in patients with chronic venous disease: San Diego population study. J Vasc Surg 37:1047-1053.

[3] Gloviczki P, Comerota AJ, Dalsing MC, Eklof BG, Gillespie DL, et all (2011) The care of patients with varicose veins and associated chronic venous diseases: Clinical practice guidelines of the Society for Vascular Surgery and the American Venous Forum. J Vasc Surg 5Suppl: 1S-48S

[4] Aleksander Sieroń, Lech Cierpka, Zbigniew Rybak, Agata Stanek. Angiology texbook. Alfa Medica Press, Bielsko Biała 2009

[5] Cholewka A, Drzazga Z, Sieroń A, Stanek A (2010) Thermovision diagnostics in chosen spine diseases treated by whole-body cryotherapy. J Therm Anal Calorim 102: 113-119.

[6] Hess CT. Identifying and managing venous dermatitis. Adv Skin Wound Care. 2005 Jun;18(5 Pt 1):242-3

[7] Bauer J, Hurnik P, Zdziarski J, Mielczarek W, Podbielska H.Thermovision and its applications in medicine. Acta BioOpt Inf Med. 1997;3(2-4):121-31.

[8]. Boerner E, Bauer J, Ratajczyk B, Deren' E, Podbielska H. Application of thermovision for analysis of superficial temperature distribution changes after physiotherapy. J Therm Anal Calorim. 2015;120(1):261-7. 